\title{
Predição de risco de óbito pelo Indice de Comorbidade de Charlson
}

\section{Prediction of risk of death with the use of the Charlson Comorbidity Index}

Rio de Janeiro, 22 de outubro de 2010

Prezados editores,

A Revista de Saúde Pública publicou um trabalho ${ }^{2}$ no qual medidas de co-morbidade foram utilizadas para a predição do risco de óbito em pacientes hospitalizados com problemas respiratórios ou cardíacos. Um dos índices aplicados para isso foi o conhecido Índice de Comorbidade de Charlson (ICC), adaptado para a Classificação Internacional de Doenças $10^{\mathrm{a}}$ Revisão (CID-10) a partir do esquema introduzido por Quan et al. ${ }^{3}$

Sem qualquer intenção de reproduzir as polêmicas Deyo vs Manitoba-Darthmouth da década de 1990, ${ }^{1,5}$ queremos ressaltar a existência de um esquema alternativo para essa adaptação. ${ }^{4}$ Como seria de esperar, as diferenças entre esses esquemas são de pouca monta, sendo mais notável, apenas, a introdução da categoria "AIDS+any tumor, including leukemia and linphoma", com peso "8" (pensada como a justaposição das duas condições). Embora, naturalmente, mais estudos sejam necessários para uma comparação adequada, e embora diferenças (se houver) tendam a ser pequenas, utilizando-se a conhecida métrica $c$ da regressão logística, o esquema ${ }^{4}$ saiu-se melhor na predição da mortalidade geral em pacientes hospitalizados ( $c=0,76$ vs 0,70$)$. De qualquer forma, esse esquema é também "uma proposta que visa adotar uma codificação padronizada de uso internacional". ${ }^{2}$

Renan MVR Almeida

Wagner Coelho de Albuquerque Pereira Universidade Federal do Rio de Janeiro renan.m.v.r.almeida@gmail.com

\section{REFERÊNCIAS}

1. Deyo RA. Adapting a clinical comorbidity index for use with ICD-9-CM administrative data: a response. J Clin Epidemiol. 1993;46(10):1081-2.

2. Martins MS. Uso de medidas de comorbidades para predição de risco de óbito em pacientes brasileiros hospitalizados. Rev Saude Publica. 2010; 44(3):44856. DOI:10.1590/S0034-89102010005000003

3. Quan H, Sundararajan V, Halfon P, Fong A, Burnand B, Luthi JC, et al. Coding algorithms for defining comorbidities in ICD-9-CM and ICD-10 administrative

\section{RESPOSTA DA AUTORA}

Rio de Janeiro, 19 de novembro de 2010

Prezados editores,

Os autores da carta ao editor destacam dois pontos

referidos com freqüência quando o tema diz respeito ao uso do ICC, ${ }^{1}$ quais sejam: adaptação aos códigos da CID-10 e revisão das condições clínicas e dos respectivos pesos. Desenvolvido empiricamente, Charlson

4. Ramiarina RA, Ramiarina BL, Almeida RMVR, Pereira WCA. Comorbidity adjustment index for the international classification of diseases, $10^{\text {th }}$ revision. Rev Saude Publica. 2008;42(4):590-7. DOI:10.1590/ S0034-89102008000400003

5. Romano PS, Roos LL, Jollis JG. Adapting a clinical comorbidity index for use with ICD-9-CM administrative data: differing perspectives. J Clin Epidemiol. 1993;46(10):1075-9. 
et $\mathrm{al}^{1}$ examinaram 30 condições clínicas para construir o índice composto por 19 dessas condições, cuja ponderação foi atribuída com base no risco relativo. Originalmente proposto para estudos longitudinais de doenças crônicas, ${ }^{1}$ vem sendo utilizado em estudos observacionais. ${ }^{5}$ As primeiras adaptações desse índice para a nona revisão da CID, com modificações clínicas (CID-9-MC), foi usada principalmente nos Estados Unidos. ${ }^{9}$ Comparações dessas traduções mostram discrepância nos códigos selecionados e o emprego desses dois métodos mostrou efeito reduzido sobre o poder de predição do risco de morrer. ${ }^{2,9}$

Considerando o debate sobre a qualidade das traduções do ICC para a CID e a inexistência na época de tradução para a CID-10, optou-se em estudo anterior por adaptar as condições clínicas tanto para a CID-9 como para a CID-10. ${ }^{3,4}$ Esse procedimento foi realizado por um pesquisador com formação médica e em codificação de doenças. Contemporânea à adaptação realizada por Martins et al ${ }^{4}$ (2006), pesquisadores suíços, australianos e canadenses desenvolveram algoritmos para tradução do ICC para a CID-10. ${ }^{7}$ Considerando as três iniciativas anteriores, Quan et $\mathrm{al}^{7}$ propuseram-se a compatibilizar e revisar as adaptações para a CID-10 disponíveis na literatura com o intuito de obter uma listagem consensual de códigos e assim desenvolver um algoritmo para as condições clínicas do Índice de Charlson. ${ }^{1} \mathrm{O}$ processo utilizado envolveu codificação independente e painel de médicos para revisão e a aplicação do algoritmo em modelos de predição de óbito apresentou uma estatística $c$ igual a $0,86 .{ }^{7} \mathrm{~A}$ adaptação do ICC para a CID-10 realizada por Martins et $\mathrm{al}^{10}$ apresenta diferenças com relação aos códigos diagnósticos empregados por Quan et al, ${ }^{7}$ especialmente o número de dígitos utilizados na codificação. Contudo, os resultados encontrados mostraram que essas diferenças não tiveram efeito significativo sobre o escore de gravidade dos pacientes e a capacidade discriminativa dos modelos de predição de óbito foi igual $(0,69)$. Apesar de a adaptação ter sido realizada por especialista na área de codificação, ${ }^{3,4}$ pode-se considerar que a abordagem metodológica realizada por Quan et $\mathrm{al}^{7,10}$ foi mais robusta, sobretudo quando se considera a complexidade inerente à CID e a expertise necessária ao seu manuseio. A estratégia adotada por Ramiarina et $a^{8}$ leva a conclusão similar. Além disso, atualmente esforços nessa linha devem ser fomentados quando aspectos muito particulares descrevem o perfil de morbidade da população de estudo - o que nem sempre ocorre.

Outro ponto levantado tem relação com o desenvolvimento empírico do ICC, a partir do qual se pode aventar a existência de insuficiências no rol de condições clínicas incluídas e inadequações no peso atribuído a cada uma. Conseqüentemente, uma dimensão avaliada do ICC requer o recálculo dos pesos (recalibragem), um procedimento que objetiva julgar a validade do uso em população com perfil demográfico e de morbi-mortalidade diferenciado da população usada para gerar essas ponderações. Alguns trabalhos sublinham a importância de gerar empiricamente pesos, particularmente quando se avalia diagnóstico ou procedimento especifico, e incluir outras comorbidades no ICC para aumentar o poder de predição de modelos de predição de risco. ${ }^{2}$ Análise nessa linha foi realizada utilizando dados brasileiros provenientes do sistema de informação sobre produção hospitalar desenvolvido pela Faculdade de Medicina de Ribeirão Preto. ${ }^{3,4}$ Contudo, o ICC com novos pesos não alterou a capacidade de discriminação do modelo quando comparado ao ICC original. Essas estratégias de revisão dos pesos contribuem para a criação de índices mais adequados à população de estudo, ao diagnóstico principal e ao resultado do cuidado. Entretanto, o desenvolvimento de um índice e novos pesos a cada estudo dificulta a comparação dos achados, é custoso e fere o princípio científico de parcimônia.

Apesar de não aventado pelos autores, um aspecto importante, sobretudo na realidade brasileira, é a necessidade de melhoria nos sistemas de informação sobre morbidade hospitalar. Obviamente há a recomendação de ampliação do número de campos para o registro completo dos diagnósticos; em outros países, os formulários permitem a codificação de diagnósticos secundários, variando entre 15 e 25 campos. Também houve iniciativas para enfrentar o desafio de distinguir complicação de comorbidade, que a princípio requer conhecimento detalhado, tanto do estado de saúde do paciente no momento de admissão quanto sobre o curso do processo de cuidados. Como, em geral, a base de dados administrativos não especifica a data de diagnóstico, alguns sistemas possuem uma variável que informa se a condição estava ou não presente na admissão hospitalar. $^{6}$ Essa informação é importante para distinguir entre doença crônica ou em curso e ajuda a identificar complicações devido à qualidade do cuidado.

Monica Martins Escola Nacional de Saúde Pública Sergio Arouca Fundação Oswaldo Cruz martins@ensp.fiocruz.br 


\section{REFERÊNCIAS}

1. Charlson ME, Pompei P, Ales KL, MacKenzie CR. A new method of classifying prognostic comorbidity in longitudinal studies: development and validation. J Chron Dis. 1987;40(5):373-83.

2. Cleves MA, Sanchez N, Draheim M. Evaluation of two competing methods for calculing Charlson's comorbidity index when analyzing short-term mortality using admistrative data. J Clin Epidemiol. 1997;50(8):903-8.

3. Martins M, Blais R, Miranda NN. Avaliação do índice de comorbidade de Charlson em internações da região de Ribeirão Preto, São Paulo, Brasil. Cad Saude Publica. 2008;24(3):643-52. DOI:10.1590/S0102311X2008000300018

4. Martins M, Blais R. Evaluation of comorbidity indices for inpatient mortality prediction models. J Clin Epidemiol. 2006;59(7):665-9. DOI:10.1016/j.jclinepi.2005.11.017

5. Needham DM, Scales DC, Laupacis A, Pronovost PJ. A systematic review of the Charlson comorbidity index using administrative databases: a perspective on risk adjustment in critical care research. J Crit Care. 2005; 20(1):12-9. DOI:10.1016/j.jcrc.2004.09.007
6. Quan H, Parsons GA, Ghali WA. Assessing accuracy of diagnosis-type indicators for flagging complications in administrative data. J Clin Epidemiol. 2004;57(4):36672. DOI:10.1016/j.jclinepi.2003.01.002

7. Quan H, Sundararajan V, Halfon P, Fong A, Burnand B, Luthi JC, et al. Coding algorithms for defining comorbidities in ICD-9-CM and ICD-10 administrative data. Med Care. 2005:43(11):1130-9.

8. Ramiarina RA, Ramiarina BL, Almeida RMVR, Pereira WCA. Comorbidity adjustment index for the international classification of diseases, $10^{\text {th }}$ revision. Rev Saude Publica. 2008;42(4):590-7. DOI:10.1590/ S0034-89102008000400003

9. Romano PS, Roos LL, JG Jollis JG. Further evidence concerning the use of a clinical comorbidity index with ICD-9-CM administrative data. J Clin Epidemiol. 1993;46(10):1085-90.

10. Sundararajan V, Quan $\mathrm{H}$, Halfon P, Fushimi K, Luthi J, Burnand B, Ghali WA. Cross-national comparative performance of three versions of the ICD-10 Charlson index. Med Care. 2007;45(12):1210-5. DOI:10.1097/ MLR.0b013e3181484347 